\title{
COVID-19 Outbreak: an Update on Therapeutic Options
}

\author{
Kalpana Panati ${ }^{1}$ (D) Venkata Ramireddy Narala ${ }^{2}$
}

Accepted: 26 March 2020 / Published online: 1 April 2020

(C) Springer Nature Switzerland AG 2020

Dear Editor,

Initial identification of novel coronavirus disease (COVID19) in Wuhan was alerted by the ophthalmologist by name Li Wenliang and after the enquiry of reporting unusual cases of pneumonia by Wuhan Municipal Health Commission geared up the panic in public. Though it was believed to be linked with Wuhan Huanan seafood wholesale market initially, now it is spread to more than 166 countries in the world, and it was declared as pandemic by WHO. Whole landscape of the disease has changed from epidemic to pandemic in just 45 days. Worldwide, 191,127 confirmed cases and 7807 deaths were reported as on March 18, 2020, indicating serious global public health concern (https://www.who.int/docs/ default-source/coronaviruse/situation-reports/20200318sitrep-58-covid-19.pdf?sfvrsn=20876712_2). Situational awareness in all levels for risk management is badly required to contain the spread of this deadly disease.

Based on SARS and MERS coronaviruses, it was believed 2-14 days as incubation period for COVID-19. The Chinese researchers found that the incubation period could be 24 days and asymptomatic carriers were evidenced to transmit the COVID-19 also. The incubation period may be as long as 0-24 days in some cases (https://www. pharmaceutical-technology.com/news/coronavirus-studyincubation-period/). The viral genomes, isolated from both bronchoalveolar lavage fluid and cell cultures, were sequenced using next-generation sequencing, and found

Kalpana Panati and Venkata Ramireddy Narala contributed equally to this work.

This article is part of the Topical Collection on COVID-19

Kalpana Panati

panatikalpana@gmail.com

1 Department of Biotechnology, Government College for Men, Kadapa, AP 516004, India

2 Department of Zoology, Yogi Vemana University, Kadapa, AP 516 005, India
COVID-19 virus was similar to betacoronavirus 'bat-SLCoVZC45'. However, the remarkable exception was the spike protein, which is only $80 \%$ similar to bat coronaviruses. The envelope spike protein plays major role in binding to receptor, membrane fusion, and transmission capacity. Interestingly, it was more similar to SARS-CoV with respect to receptor-binding domain [1]. Despite there are some variations in COVID-19 receptor-binding domain, it was suggested that COVID-19 might use angiotensin-converting enzyme 2 (ACE2) as cell membrane receptor similar to SARS-CoV [1]. ACE2 is expressed by epithelial cells of the lung, intestine, kidney, and blood vessels.

At present, no specific antiviral treatment and no efficient vaccines are available for COVID-19 in humans. Recently, two familiar broad-spectrum antiviral drugs remdesivir and favipiravir have been tested against clinical isolate of COVID-2019 in vitro. They found that $\mathrm{EC}_{90}$ of a remdesivir, an adenosine analogue, as $1.76 \mu \mathrm{M}$ which can be achieved in non-human primate studies. Moreover, their preliminary data showed that it inhibits COVID-19 virus infection also in human liver cancer (Huh-7) cells.

Some clinical trials with remdesivir, ritonavir-boosted lopinavir monotherapy, have been launched (ChiCTR2000029308, NCT04257656). Xia et al. developed a pan-CoV fusion inhibitor, EK1 peptide, to inhibit the infection of five human coronaviruses including SARS-CoV. The same group showed that the EK1 peptide and the peptide derived from HR2 domain in spike protein of COVID-19 virus could effectively inhibit COVID-19 pseudo virus infection and domain S2 mediated cell fusion [2]. It has been recently shown by using probative assays that SARS-CoV receptor-binding domain-specific monoclonal antibody, CR3022, could potentially bind with COVID-19 viral receptor-binding domain.

There are some medical conditions, which are being treated by ACE inhibitors and angiotensin II type-I receptor blockers, such as type- 1 and type- 2 diabetes and hypertension that result in the increased expression of ACE2 [3]. It was hypothesised that treatment with ACE2-stimulating 
drugs may facilitate the infection with COVID-19 virus and increase the risk of developing severe fatal COVID19 viral infection [4]. The ACE2 gene polymorphisms might play important role in the binding of viral spike protein to cell membrane in turn COVID-19 viral infection. It suggests that the recovery of an individual is based on both therapy and ACE2 polymorphism.

Chloroquine, an antimalarial drug, has been reported as potential broad-spectrum antiviral drug, and it also has shown to inhibit viral infection of SARS-CoV. In a recent study, it was shown that chloroquine efficiently inhibits the COVID-19 viral infection in Vero E6 cells at entry and post-entry stages [5]. As chloroquine is cheap and safe drug and is being used for last 70 years, it can be clinically used against COVID-19. Further studies are warranted to better understand its efficacy.

Effective cure in the form of vaccine or therapeutic drug is the need of hour to control the COVID-19 from infecting more people. Many vaccine candidates for COVID-19 are in preclinical stage (https://www.who.int/blueprint/prioritydiseases/key-action/novel-coronavirus-landscape-ncov.pdf? ua=1). The vaccine candidate developed against SARS-CoV should be evaluated against COVID-19 virus for its efficacy in inducing neutralizing antibodies as there is similarity with respect to spike protein.

Funding information This work was supported by the Science \& Engineering Research Board (SERB) (EMR/2017/000973) Department of Science \& Technology, Government of India.

\section{Compliance with Ethical Standards}

Conflict of Interest The authors declare that they have no conflict of interest.

\section{References}

1. Lu R, Zhao X, Li J, Niu P, Yang B, Wu H, et al. Genomic characterisation and epidemiology of 2019 novel coronavirus: implications for virus origins and receptor binding. Lancet. 2020;395(10224): 565-74.

2. Xia S, Zhu Y, Liu M, Lan Q, Xu W, Wu Y, et al. Fusion mechanism of 2019-nCoV and fusion inhibitors targeting HR1 domain in spike protein. Cell Mol Immunol. 2020. https://doi.org/10.1038/s41423020-0374-2.

3. Li XC, Zhang J, Zhuo JL. The vasoprotective axes of the reninangiotensin system: physiological relevance and therapeutic implications in cardiovascular, hypertensive and kidney diseases. Pharmacol Res. 2017;125(Pt A):21-38.

4. Fang L, Karakiulakis G, Roth M. Are patients with hypertension and diabetes mellitus at increased risk for COVID-19 infection? Lancet Respir Med. 2020. https://doi.org/10.1016/S2213-2600(20)30116-8.

5. Wang M, Cao R, Zhang L, Yang X, Liu J, Xu M, et al. Remdesivir and chloroquine effectively inhibit the recently emerged novel coronavirus (2019-nCoV) in vitro. Cell Res. 2020;30(3):269-71.

Publisher's Note Springer Nature remains neutral with regard to jurisdictional claims in published maps and institutional affiliations. 